\title{
Growth-rate-dependent dynamics of a bacterial genetic oscillator
}

\author{
Matteo Osella ${ }^{1,2, *}$ and Marco Cosentino Lagomarsino ${ }^{1,2,3}$ \\ ${ }^{1}$ Genomic Physics Group, UMR7238 CNRS “Microorganism Genomics”, 15, rue de l'École de Médecine, Paris, France \\ ${ }^{2}$ University Pierre et Marie Curie, 15, rue de l'École de Médecine, Paris, France \\ ${ }^{3}$ Dipartimento di Fisica, University of Torino, Via P. Giuria 1, Torino, Italy
}

(Received 3 October 2012; revised manuscript received 22 December 2012; published 30 January 2013)

\begin{abstract}
Gene networks exhibiting oscillatory dynamics are widespread in biology. The minimal regulatory designs giving rise to oscillations have been implemented synthetically and studied by mathematical modeling. However, most of the available analyses generally neglect the coupling of regulatory circuits with the cellular "chassis" in which the circuits are embedded. For example, the intracellular macromolecular composition of fast-growing bacteria changes with growth rate. As a consequence, important parameters of gene expression, such as ribosome concentration or cell volume, are growth-rate dependent, ultimately coupling the dynamics of genetic circuits with cell physiology. This work addresses the effects of growth rate on the dynamics of a paradigmatic example of genetic oscillator, the repressilator. Making use of empirical growth-rate dependencies of parameters in bacteria, we show that the repressilator dynamics can switch between oscillations and convergence to a fixed point depending on the cellular state of growth, and thus on the nutrients it is fed. The physical support of the circuit (type of plasmid or gene positions on the chromosome) also plays an important role in determining the oscillation stability and the growth-rate dependence of period and amplitude. This analysis has potential application in the field of synthetic biology, and suggests that the coupling between endogenous genetic oscillators and cell physiology can have substantial consequences for their functionality.
\end{abstract}

DOI: 10.1103/PhysRevE.87.012726

PACS number(s): 87.17.Aa, 87.18.Vf, 87.16.Yc, 82.40.Bj

\section{INTRODUCTION}

Oscillatory behavior is widespread and fundamentally important in biological systems, from circadian clocks to cellcycle control [1]. At the level of a single cell, oscillations can be sustained by regulatory circuits, the basic components of which are nucleic acids, proteins, and biochemical interactions $[2,3]$. However, naturally occurring regulatory systems are complex. Thus, our path to rationalize their behavior has to pass through simplifications and basic underlying principles [4]. The study of minimal circuits, by mathematical modeling and experimental synthetic engineering, allows a quantitative approach to the study of gene expression [5-8].

This simplifying approach is not only useful for studying the extant regulatory circuits. Understanding the basic principles and dynamical properties of regulatory networks makes it possible to design and produce synthetic circuits that can perform specific functions in a predictable manner [9-12], with potentially relevant future biotechnological and biomedical applications [11,13]. A paradigmatic example of a synthetic genetic oscillator, and one of the earliest in vivo realizations [14], is the so-called "repressilator," a three-gene cyclic circuit where each gene protein product represses the synthesis of its successor [Fig. 1(a)].

From a physics standpoint, it is desirable to build minimal but effective and testable models, with qualitative and possibly quantitative predictive power for experiments. To this end, dealing with controlled, well-characterized, and isolated systems is necessary. Unfortunately, every genetic circuit, endogenous or synthetically implemented in a living cell, can not be truly considered isolated from cellular processes. These

*Corresponding author: matteo.osella@upmc.fr processes are strongly affected by the physiological state of the cell [15], and thus by the environmental conditions and type and availability of nutrients. For example, the macromolecular composition of bacterial cells in "steady exponential" growth (i.e., constantly dividing at the same rate ${ }^{1}$ ) changes substantially with growth rate (the rate of cell proliferation) [16-18]. Importantly, in this case, growth rate appears to encapsulate most of the physiological changes. In other words, cells growing on different nutrients but at similar growth rates are in many ways equivalent, considering important global cell parameters such as concentrations of ribosomes and RNA polymerases (the molecular machines effecting translation and transcription, respectively). Such growth-rate-dependent parameters affect gene expression, coupling its dynamics with the cell state, with relevant consequences for genetic circuit functioning $[15,19,20]$.

The emergent quantitative "laws" of bacterial physiology, linking cell composition and growth rate, are reminiscent of those of thermodynamics, and were the subject of recent advances using physical modeling $[17,18]$. Previously, they were in part captured by early studies in the 1950s and 1960s [16]. However, to date, they are not completely characterized. For E. coli, the best studied bacterial species, the growth-rate dependencies of various cellular parameters were evaluated phenomenologically in a study by Klumpp and co-workers [15], compiling results from multiple experiments. Leveraging on these empirical data, they analyzed the growth-rate dependence of the steady state protein concentration of a constitutively expressed (i.e., unregulated) gene and few other

\footnotetext{
${ }^{1}$ This state is conventionally referred to as "steady" because its maintenance requires that a nutrient sink (the cells) is constantly replenished with a source.
} 
simple genetic circuits, such as the self-regulator and the toggle switch. Their modeling strategy will be briefly reviewed in Sec. II A. Beyond steady state, knowing the empirical growth-rate dependence of gene expression parameters allows us in principle to revisit the dynamical properties of genetic circuits, introducing the physiological cell state as a new player.

This work addresses the growth-rate dependence of a biological oscillator dynamics, and considers the effect of cell state on a repressilator, integrated on either a plasmid or the E. coli chromosome. From the physical modeling viewpoint, this entails understanding the models and phenomenological laws that relate the circuit parameters to the cell physiological state. Incorporating these features in otherwise fairly standard models leads to predictions that are experimentally relevant. After reviewing the conditions for oscillations and the repressilator dynamics at fixed-growth rate (and extending some known results), we will show how the physiology of the cell can alter qualitatively and quantitatively the dynamical properties of the system. The main result is that the conditions for stable oscillations, as well as their amplitude and period are growthrate dependent. This implies that a genetic oscillator can display distinct dynamical behaviors in different environments and nutrient conditions. Additionally, the circuit support, e.g., type of plasmid or chromosomal position of the genes, also contributes to its dynamics, through gene dosage. Specifically, the range of growth rates in which oscillations are observable will vary with support, in a manner that could be exploited both biologically and technologically. Our predictions, although based on a simplified model, are experimentally testable in a straightforward way on synthetically realized repressillator circuits, and more in general set a framework for the more complex case of endogenous oscillators in bacteria, prominently the cell cycle and circadian clocks [21].

The paper is organized as follows. Section II reviews the modeling strategy adopted to include the growth rate as a variable in gene expression (Sec. II A) and explains how this strategy can be applied to a mathematical description of the repressilator (Sec. IIB). The Appendix reviews the Cooper-Helmstetter model [22], an empirical model of DNA replication in fast-growing bacteria that is an essential part of our approach, and justifies in more detail some of the model assumptions presented in Sec. II. Section III contains the quantitative analysis of the circuit, and in particular of the role played by the cellular state of growth on the dynamics. More specifically, Sec. III A analyzes the symmetric repressilator, showing the possibility of a growth-mediated switch between oscillations and convergence to a stable fixed point. Section III B focuses on the asymmetric case, with particular emphasis on the role of gene chromosomal position on the circuit behavior. Finally, the last section discusses the implications of the results from both a biological and a physical modeling standpoint.

\section{MODEL}

\section{A. Growth-rate dependence of gene expression}

This section reviews the approach of Klumpp and coworkers [15] and the necessary assumptions in order to extend it to the analysis of circuit dynamics. This modeling strategy constitutes the basis for our description of the repressilator, introduced in Sec. II B.

In absence of regulation, the dynamics of messenger RNA (mRNA) levels and protein concentrations, denoted with $m$ and $p$, respectively, is described by two equations

$$
\dot{m}=\frac{g \alpha_{m}}{V}-\beta_{m} m, \quad \dot{p}=\alpha_{p} m-\beta_{p} p,
$$

where $\alpha_{m}$ and $\alpha_{p}$ are the transcription and translation rates, respectively, $\beta_{m}$ and $\beta_{p}$ the degradation rates of mRNAs and proteins, and $V$ the cell volume. The parameter $g$ is the gene copy number. If the gene support is a plasmid, $g$ represents the (mean) plasmid copy number. For genes integrated on the chromosome, the copy number can vary because of DNA replication, especially at fast growth rates, when multiple copies of the genome are replicated at the same time. This phenomenon whereby gene dosage is modified by DNA replication is described by the Cooper-Helmstetter model [22], and reviewed in the Appendix. Note that for fast $E$. coli growth, $g$ increases with decreasing distance $\ell$ from the replication origin [illustrated in Fig. 1(b)] since the cell engages overlapping rounds of DNA replication in order to allow fast growth [23].

In bacteria, proteins are typically stable, with a lifetime longer than the cell cycle, while mRNAs have a lifetime of just a few minutes [24]. Therefore, the loss of protein is mainly due to dilution through growth and cell division, so that an effective degradation rate $\beta_{p}=\mu \ln 2$ (where $\mu$ is the growth rate) can be safely used in most cases. On the other hand, the fast time scale of mRNA dynamics allows a quasiequilibrium approximation. Thus, Eq. (1) can be reduced to

$$
\dot{p}=\frac{g \alpha_{m} \alpha_{p}}{\beta_{m} V}-\beta_{p} p
$$

Rescaling time with the dilution rate $\beta_{p}$, all the growth-rate dependence can be factorized in a single term $F(\mu)$ :

$$
\dot{p}=\frac{g \alpha_{m} \alpha_{p}}{\beta_{m} \beta_{p} V}-p=p^{*} F(\mu)-p .
$$

Normalizing the growth-rate dependence $F(\mu)$ such that $F(\mu)=1$ for $\mu=1 \mathrm{db} / \mathrm{hr}$ (i.e., doublings per hour), the parameter $p^{*}$ represents the steady-state concentration of the constitutive (unregulated) gene at $\mu=1 \mathrm{db} / \mathrm{hr}$. Thus, it is a measure of the degree of basal expression. Klumpp and coworkers [15] refer to this quantity as the "promoter strength." However, this terminology might be slightly misleading, as the term actually includes nontranscriptional parameters, such as the translation efficiency or the gene copy number at $\mu=1 \mathrm{db} / \mathrm{hr}$. Thus, we will refer to it as the "basal expression" level in the following.

In principle, all the cellular parameters in $F(\mu)$ may display a growth-rate dependence. The volume $V$ is known experimentally to change with growth rate [16,25], with faster-growing bacteria being larger than slower-growing ones. The protein degradation rate $\beta_{p}$ is a direct consequence of dilution due to cell growth and division, at least for stable proteins, and therefore, as previously discussed, it is a linear function of the growth rate. By contrast, the empirical growth-rate independence of the mRNA degradation rate $\beta_{m}$ has a less obvious interpretation [15]. In order to sustain 
fast growth and a large cell mass, the cellular abundance of the transcriptional and translational machinery is expected to increase at fast growth, and indeed this is what can be observed experimentally [16]. However, the rates of transcription and translation $\left(\alpha_{m}\right.$ and $\left.\alpha_{p}\right)$ of a gene only reflect the availability of "free" (active but not already engaged in any process) RNA polymerases and ribosomes. This fraction can be quite hard to estimate [26], requiring several empirical measurements, and is in general not merely proportional to the total abundance. In fact, while the cellular ribosome content increases strongly with growth rate, the translation rate is apparently constant in different nutrient conditions [15]. The gene dosage $g$ is probably the best-characterized parameter since it is captured by the Cooper-Helmstetter model [22]. Its increase with growth rate is a consequence of the coupling between DNA replication and growth. To sum up, while it is possible to try to rationalize at least some of the growth-rate dependencies of the basic parameters, there is in general no a priori quantitative expectation (and sometimes not even a basic intuition) for them, and thus for $F(\mu)$. Hence, $F(\mu)$ can be fully defined only through the empirical knowledge of the growth-rate dependencies of the different parameters.

For E. coli, the numerical values of these parameters were collected for five growth rates $\mu$ between 0.6 and $2.5 \mathrm{db} / \mathrm{hr}$ [15]. These values are reported in the Supplemental Material of Klumpp et al. (Ref. [15]), more specifically in Table S1. The growth-rate dependencies of the various parameters are plotted in Fig. 1 of the same paper. As a combination of these parameters, $F(\mu)$ decreases in a weakly nonlinear fashion in the available range of growth rates (interpolation will be used in the following when needed). The growth-rate dependence of $F(\mu)$, and thus of the concentration of the protein product of a constitutive gene, is reported in Fig. 2 D and in Fig. 3 of the same Ref. [15].

The empirical growth-rate dependencies that define $F(\mu)$ are based on experimental measurements of the average cellular properties in a growing cell population. Thus, they are in principle also dependent on the age distribution (where "age" stands for stage of the cell cycle) of the population. However, the differences between averages calculated over a cell cycle and over a cell population with the age distribution determined by the exponential growth are not quantitatively significant, as discussed in detail in the Appendix. Therefore, the available empirical measurements of all the key parameters, which are based on population averages, will be used throughout this paper as an approximation of their cell-cycle averages.

Moreover, we will not consider explicitly the cell-cycle dynamics of cellular parameters, which, for sufficiently long time scales, can be averaged out using for example effective gene dosage or effective protein dilution rate. As a consequence, Eq. (3) can accurately describe the dynamics of protein concentration on time scales longer than the generation time [27], as it will always be the case in the following.

\section{B. The repressilator}

The repressilator is a genetic network consisting of three genes, each of which encodes for a "transcription factor" (i.e., a protein able to control the transcription level of one or (a)

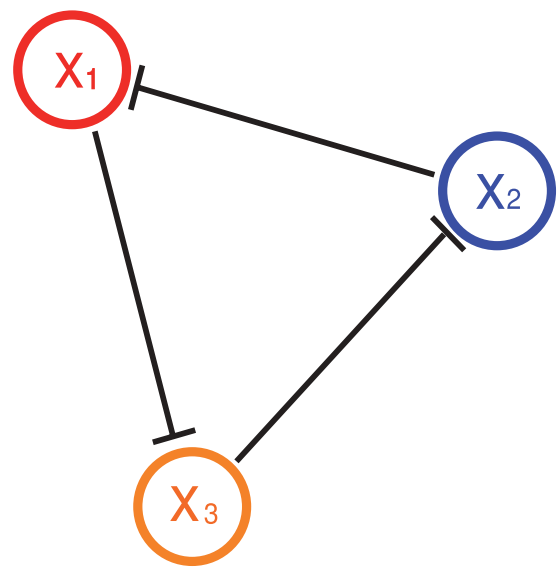

(b)

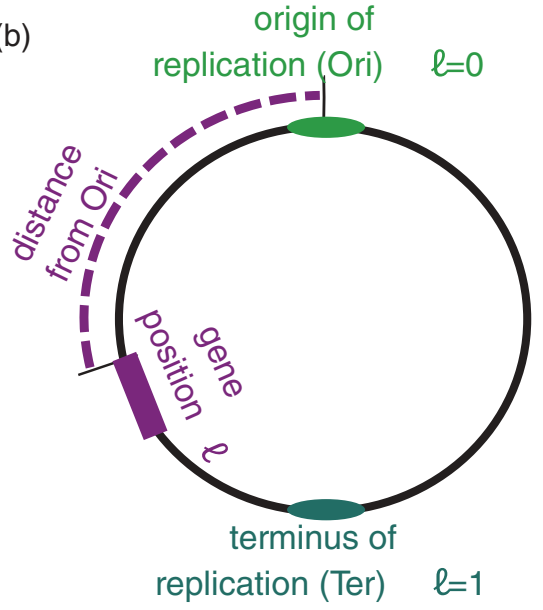

FIG. 1. (Color online) (a) Scheme of the repressilator. Each node represents the expression level of one gene, links represent repressive regulatory interactions, as described by Eq. (4). (b) Scheme of the $E$. coli chromosome, illustrating the coordinate $\ell$, used for the positions where a gene can be inserted.

a set of target genes by binding to their upstream genomic region) that represses the expression of its target in a cycle of regulations [Fig. 1(a)]. The effect of this negative regulation can be modeled phenomenologically as a multiplicative factor rescaling the target production rate [the positive term on the right-hand side of Eq. (3)] with a nonlinear function of the repressor concentration (called "Hill function") $[5,6]$ :

$$
R(x / k)=\frac{1}{1+(x / k)^{n}},
$$

where the Hill coefficient $n$ defines the degree of cooperativity (determining the steepness of $R$ ), while the dissociation constant $k$ specifies the repressor concentration at which the production rate is half of its constitutive value. With this mathematical representation of transcriptional regulation, the repressilator circuit can be described by three equations, one for each gene, based on the gene expression model discussed in the previous section:

$$
\begin{aligned}
& \dot{x_{1}}=x_{1}^{*} F_{1}(\mu) R\left(x_{2} / k_{1}\right)-x_{1}, \quad \dot{x_{2}}=x_{2}^{*} F_{2}(\mu) R\left(x_{3} / k_{2}\right)-x_{2}, \\
& \dot{x_{3}}=x_{3}^{*} F_{3}(\mu) R\left(x_{1} / k_{3}\right)-x_{3},
\end{aligned}
$$


where the terms $x_{i}^{*}$ are the basal expression levels. They represent the steady-state concentration of protein $i$ in absence of repression at $\mu=1 \mathrm{db} / \mathrm{hr}$. Therefore, the "basal expression level," as defined here for a negatively regulated gene, is the maximal expression level that can be achieved in a fixed growth condition $(\mu=1 \mathrm{db} / \mathrm{hr})$ in absence of regulation. ${ }^{2}$

The growth-rate functions $F_{i}(\mu)$ can be gene specific due to the dependence of gene dosage $g_{i}$ on the chromosomal gene position. Assuming similar dissociation constants for the three promoters $k_{1} \simeq k_{2} \simeq k_{3}=k$, the protein concentrations can be rescaled with $k$ :

$$
\begin{aligned}
& \dot{x_{1}}=x_{1}^{*} F_{1}(\mu) R\left(x_{2}\right)-x_{1}, \quad \dot{x_{2}}=x_{2}^{*} F_{2}(\mu) R\left(x_{3}\right)-x_{2}, \\
& \dot{x_{3}}=x_{3}^{*} F_{3}(\mu) R\left(x_{1}\right)-x_{3} .
\end{aligned}
$$

With these notations, the basal expression levels $x_{i}^{*}$ have dimensionless units, as they are protein concentrations rescaled with the dissociation constant $k$.

\section{RESULTS}

\section{A. Growth rate affects qualitatively and quantitatively the dynamics of a symmetric repressilator}

We start the analysis from the simplified case of a symmetric repressilator, i.e., the three genes have approximately the same production and degradation rates for mRNA and proteins, as well as the same growth-rate dependence of parameters (this is attained for example when all genes are integrated on a single plasmid or at a similar distance from the origin of replication on a chromosome). This is the most studied case, and was approached with different mathematical descriptions [4,28-30]. The symmetric approximation was originally proposed to explain the behavior of the synthetic realization in vivo [14].

In the symmetric case, the functions encoding the growthrate dependence $F(\mu)$ and the basal expression levels $x^{*}$ are exactly the same for each of the three genes, simplifying Eq. (6) to

$$
\dot{x}_{i}=x^{*} F(\mu) R\left(x_{i+1}\right)-x_{i},
$$

where $i \in[1,3]$ and $x_{4} \equiv x_{1}$.

\section{At fixed growth rate, the dynamics is determined by basal expression and cooperativity}

Before addressing the effects of the growth-rate dependence of parameters, we characterize the circuit dynamics at fixed growth conditions, for simplicity at $\mu=1 \mathrm{db} / \mathrm{hr}$ where $F(\mu)=1$. In general, the symmetric repressilator can display stable oscillations, arising through a Hopf bifurcation [28]. More specifically, Eq. (7) have an oscillatory solution if the condition for cooperativity $n>2$ is satisfied, as can be shown in a straightforward way considering the symmetry of the system [31].

For a given steepness of the repression function satisfying the condition $n>2$, the stability of the limit cycle is solely

\footnotetext{
${ }^{2}$ Note that the term "basal expression" is sometimes found in the literature with a different meaning, i.e., the low but detectable level of expression that a negatively regulated gene maintains in conditions of strong repression (also called "leakage" level of expression).
}

determined by the basal expression $x^{*}$. The values of $x^{*}$ that ensure stability of the oscillatory state can be calculated using linear stability analysis and the resulting stability diagram is shown in Fig. 2. Essentially, an increase of either cooperativity or basal expression can help the stabilization of oscillations. This result is common to all the different repressilator descriptions proposed in the literature [4].

Furthermore, the two parameters $n$ and $x^{*}$ determine the amplitude and period of oscillation (Fig. 3). The approximately linear and logarithmic dependencies of amplitude and period, respectively, that emerge from numerical integration of Eq. (7) can be rationalized by the following rough but conceptually simple argument. Each gene in the repressilator tends to oscillate between two states corresponding to its maximally repressed state and its maximally activated state. In a simplified situation of a step repression function $(n \gg 1)$ that switches the target gene on and off after its equilibration, the protein concentration would go from $x_{i} \simeq 0$ (fully repressed) to $x_{i} \simeq x^{*}$ (fully activated), thus leading to a linear dependence on basal expression of the oscillation amplitude. On the other hand, the oscillation period depends on the time scales of gene activation and deactivation. For example, the time $\tau$ required to go from the fully activated state $x_{i}(t) \simeq x^{*}$ to $x_{i}(t) \simeq 1$, when, assuming a steplike repression function, it releases the repression of its target, is given by $x^{*} e^{-\tau}=1 \rightarrow$ $\tau=\ln \left(x^{*}\right)$. This expression suggests that the basal expression contributes logarithmically to the deactivation time scale, which is compatible with the dependence of the period on $x^{*}$ measured by numerical integration shown in Fig. 3(b).

\section{Increasing the growth rate can destabilize oscillations}

Let us now consider a specific experimental realization of the repressillator, which uses a set of genetic components with some intrinsic parameters. Given the basal expression level and the cooperativity of repression, defined by the specific properties of the actual genes and promoters implementing the repressilator, the growth-rate dependence of parameters defines a vertical path in the stability diagram of Fig. 2 since the "effective" basal expression $F(\mu) x^{*}$ decreases nonlinearly with growth rate. This path can cross the border of stability of the limit cycle, defining a maximum growth rate at which stable oscillations can be sustained. More precisely, the basal expression level $x^{*}$ defines the position of the path at $\mu=1$ $\mathrm{db} / \mathrm{hr}$, while the function $F(\mu)$ is related to its length. In fact, given the experimentally accessible growth rates, $F(\mu)$ identifies the span of effective basal expressions that can be explored changing the growth rate, and thus the upper and lower bounds of the path. Therefore, both factors contribute to establish the range of growth rates in which oscillations are expected experimentally.

Remarkably, the circuit can show qualitatively different dynamics depending on growth rate, and hence on nutrient conditions. Figure 4 shows this for the case of cooperativity $n=3$. Sustained oscillations can be observed at slow growth, while in fast-growth conditions the dynamics can converge to a stable fixed point. The parameter range where oscillations are stable depends on where the repressilator is integrated through the gene dosage factor $g$ appearing in $F(\mu)$ [see Eq. (3)]. 


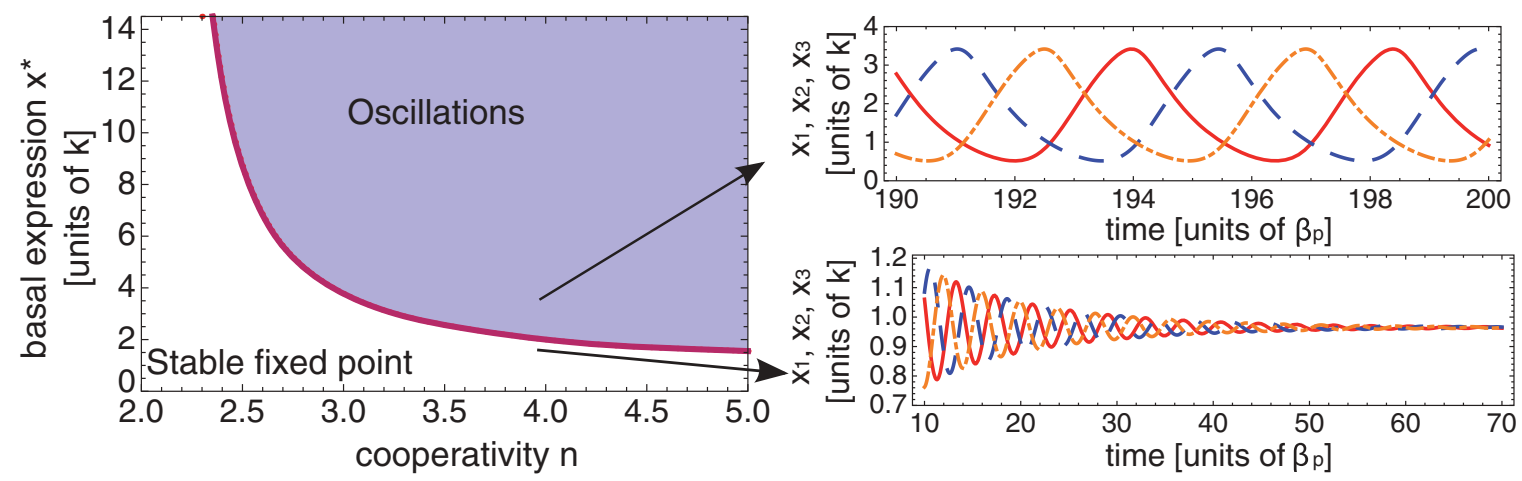

FIG. 2. (Color online) Stability diagram of the symmetric repressilator. The left panel shows the parameter space giving rise to oscillations. The basal expression $x^{*}$ is dimensionless, measured as protein concentration at $\mu=1 \mathrm{db} / \mathrm{hr}$ in absence of repression in units of the dissociation constant $k$. High repression cooperativity and high basal expression levels ("strong promoters") can ensure stable oscillation. The right panel plots are illustrative examples of the dynamics in correspondence with two sets of parameter values $\left(\left\{n=4, x^{*}=1.8\right\}\right.$ and $\left.\left\{n=4, x^{*}=5\right\}\right)$, showing the time evolution of the three protein concentrations $x_{1}, x_{2}, x_{3}$ (in units of $k$ ). The red continuous curve describes the dynamics of $x_{1}$, the blue dashed curve $x_{2}$, and the orange dotted-dashed curve $x_{3}$.

If the repressilator is integrated on a plasmid, as the original in vivo experimental realization [14], the gene dosage $g$ is simply given by the plasmid copy number, which has a plasmid-specific growth-rate dependence that can be quite
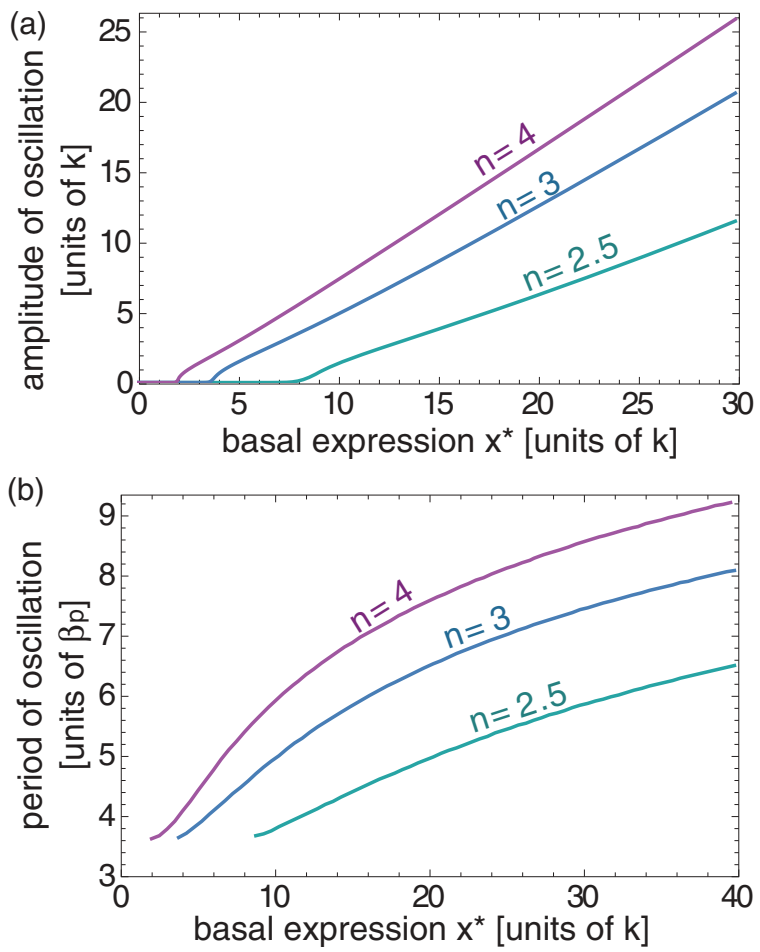

FIG. 3. (Color online) Amplitude and period of oscillation of a symmetric repressilator. The plots show oscillation amplitude (a) and period (b) as a function of the basal expression level for three different values of cooperativity $n$. Both quantities are plotted in rescaled (dimensionless) units. The amplitude (concentration) is rescaled by the dissociation constant $k$, while the period has units of protein degradation rate $\beta_{p}$. For each value of $n$, Eq. (7) were integrated numerically with values of $x^{*}$ in the range $0.1-40$ and step size 0.1 . For each numerical solution, the oscillation amplitude and period were evaluated after convergence to a stable limit cycle. strong [15,32]. Figure 4 compares the parameter regions corresponding to convergence to stable oscillation or to a fixed point for a repressilator integrated on the two plasmids $R 1$ and $p B R 322$ for which the copy number has been measured in different growth media [15].

The dosage of a chromosomal gene, instead, is determined by its genomic position as set by the Cooper-Helmstetter model [see the Appendix and Fig. 1(b)]. Therefore, for a repressilator integrated on the chromosome, the normalized circuit distance $\ell$ from the replication origin (Ori) defines the growth-rate dependence of the dynamics [where $\ell=0$ represents Ori and $\ell=1$ the replication terminus Ter, Fig. 1(b)]. It should be noticed that the three genes composing the repressilator are assumed here to be inserted approximately in a single chromosomal location [or equivalently in different replichores, the oppositely replicated chromosome halves, but at the same

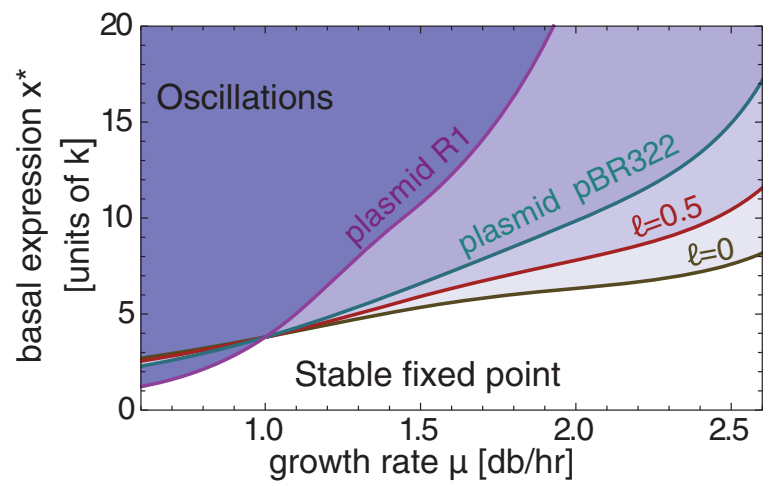

FIG. 4. (Color online) The repressilator has different dynamics in different growth conditions. At fixed repression cooperativity ( $n=3$ in this plot), the repressilator can show stable oscillation or convergence to a steady state depending on the cell growth rate $\mu$ and the basal expression $x^{*}$ (in units of $k$ ). The circuit physical support (plasmid, chromosome) defines the range of growth rates at which a limit cycle can be observed. The cases of circuit integration on plasmids $(R 1$ and $p B R 322)$ and on chromosome near the origin of replication $(\ell=0)$ and between the origin and the terminus $(\ell=0.5)$ are represented in the plot. 

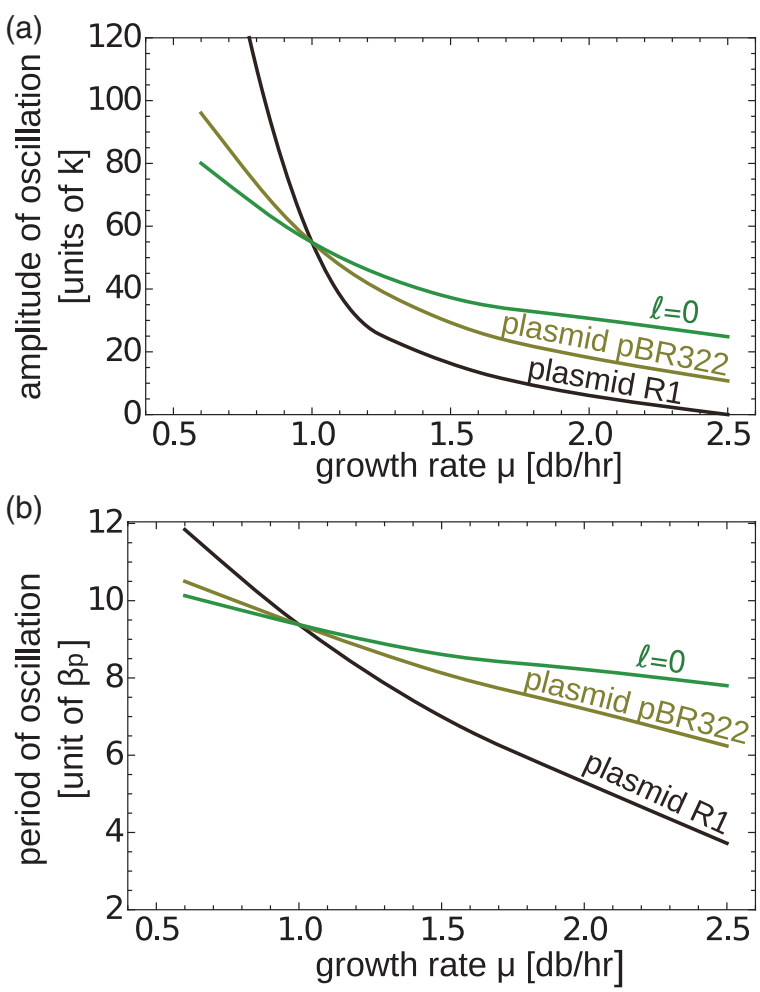

FIG. 5. (Color online) The oscillation amplitude and period of a symmetric repressilator vary significantly with growth rate. The plots show the scaling with growth rate of oscillation amplitude (concentration in units of $k$ ) and period (time in units of protein degradation rate) for a "strong promoter" (basal expression $x^{*}=70$ in $k$ units) and cooperativity $n=3$. The three curves correspond to integration of the repressilator on plasmid $R 1$, plasmid $p B R 322$, and to chromosomal integration of the three genes near the origin of replication $(\ell=0)$. A repressilator located near the replication terminus $(\ell=1$, not shown) follows a growth-rate dependence of amplitude and period similar to the case of plasmid $p B R 322$.

distance $\ell$ from Ori, see Fig. 1(b)]. The effects of the imbalance in gene dosage generated by different gene locations on the chromosome are explored in Sec. III B2.

\section{Period and amplitude of oscillation are growth rate dependent}

Figure 4 shows that increasing the basal expression $x^{*}$ (which we recall also includes parameters not based on transcription) and the steepness of the repression function $n$ can make the limit cycle solution stable in a wide range of conditions. However, even if a repressilator is designed to exhibit oscillations in the experimentally accessible conditions, the growth rate is still expected to influence the oscillation amplitude and period in a measurable way [i.e., by a factor that can exceed 5 (Fig. 5)]. Indeed, the effective basal expression $F(\mu) x^{*}$ decreases with increasing growth rate because of the functional form of $F(\mu)$, leading to a reduced amplitude and period of oscillation. Integrating Eq. (7) numerically for parameter values corresponding to different growth rates allows us to predict the oscillation period and amplitude for a chromosomally integrated repressilator or for a plasmid implementation, if the scaling of the plasmid copy number with growth rate is known. The presence of nonlinearities in the system makes the dependence on growth rate of these two variables nontrivial, as represented in Fig. 5.

\section{B. Effect of intrinsic and position-induced asymmetry on the repressilator dynamics}

Differences in intrinsic properties of the genes composing the circuit, such as affinity for RNA polymerase or ribosome binding, or gene dosage imbalances due to the specific gene location on the chromosome, lead to unequal parameter values in the equations for the dynamics of $x_{i}$. This situation is generally referred to as "asymmetric". A certain degree of asymmetry is expected for generic synthetic realizations of the repressilator. However, with the exception of a few studies $[29,33]$, its consequences on the dynamics have not been fully characterized theoretically [4]. In the modeling framework adopted here, intrinsic gene properties are summarized by the basal expression level. Additionally, using the CooperHelmstetter model (see the Appendix), it is possible to account for the position-dependent scaling of gene dosage with growth rate.

The two possible contributions to repressilator asymmetry, intrinsic gene properties and gene dosage, and their effects on the dynamics can be analyzed separately. We first address the dynamics at fixed growth rate of a repressilator composed of genes with different basal expression levels, and subsequently explore the consequences of position-induced asymmetry at different growth rates for a repressilator made of genes with equal intrinsic properties, but different chromosomal location.

\section{Effects of asymmetry at fixed growth rate}

We consider the simplified situation in which only one of the genes of the repressilator differs from the others in its basal expression by a factor $A$, but the three genes share the same growth-rate dependence $F(\mu)$. Thus, the single additional parameter $A$ introduced in the model measures the level of circuit asymmetry. The system of equations describing the dynamics of a repressilator designed this way is

$$
\begin{aligned}
& \dot{x_{1}}=x^{*} F(\mu) R\left(x_{2}\right)-x_{1}, \quad \dot{x_{2}}=x^{*} F(\mu) R\left(x_{3}\right)-x_{2}, \\
& \dot{x_{3}}=A x^{*} F(\mu) R\left(x_{1}\right)-x_{3} .
\end{aligned}
$$

At fixed growth rate [for simplicity we take the case of growth rate $\mu=1 \mathrm{db} / \mathrm{hr}$, where $F(\mu)=1$ ], linear stability analysis can be applied to study the fixed point stability. As in the symmetric case described above, a Hopf bifurcation stands between the system convergence to a stable fixed point and the oscillatory solution. For each repression cooperativity level $n$, the stability diagram can be drawn as a function of the basal expression $x^{*}$ and the asymmetry level $A$, as shown in Fig. 6 for $n=3$. This diagram essentially shows that a high degree of asymmetry destabilizes the oscillations. Therefore, if the goal is to engineer a stable oscillator, a roughly symmetric design is generally preferable.

However, the minimum of the boundary curve between the two asymptotic dynamical behaviors (continuous purple curve in Fig. 6) does not correspond to the symmetric case $\left[\log _{10}(A)=0\right]$. This result indicates that a symmetric system showing damped oscillations (as it is generally the case for parameter values just below the boundary in Fig. 6) can be 

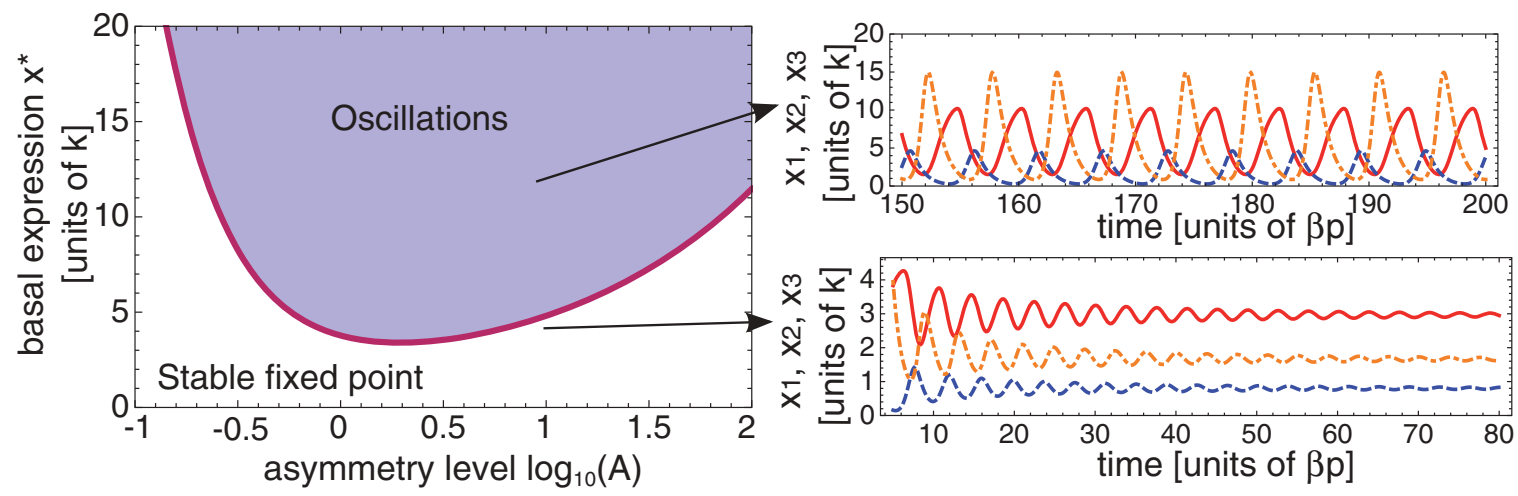

FIG. 6. (Color online) Stability diagram of the asymmetric repressilator. The parameter space is divided in the regions corresponding to convergence of the dynamics to a limit cycle and to a stable fixed point. The two parameters considered are the basal expression $x^{*}$ (in units of $k$ ) and the level of asymmetry $A$ (the factor by which the basal expression of one of the genes differs from the two others). The cooperativity is fixed to $n=3$, but the qualitative shape of the phase diagram holds for cooperativity values $n>2$. While a high degree of asymmetry reduces the robustness of the oscillatory state, a slight asymmetry can stabilize the damped oscillations showed by the symmetric system. The right panel shows an example of the dynamics of the three protein concentrations for two sets of parameter values $\left(\left\{x^{*}=12, A=10\right\}\right.$ and $\left.\left\{x^{*}=4.5, A=10\right\}\right)$. The red continuous curve describes the dynamics of $x_{1}$, the blue dashed curve refers to $x_{2}$, and the orange dotted-dashed to $x_{3}$.

pushed toward a stable oscillation regime by slightly increasing the basal expression level of just one gene, if the resulting asymmetry is not too strong.

Moreover, the presence of a gene with a different basal expression breaks the symmetry in the dynamics of protein concentrations, making the oscillation amplitude (or the stable fixed point) gene specific, as shown in Fig. 6 for two parameter sets. Thus, a possible test of the effective symmetry of experimental repressilator realizations would entail measuring the oscillation amplitude of two fluorescently tagged protein products of genes in the circuit. More specifically, the level of asymmetry, introduced by the presence of a gene with different intrinsic properties, affects the oscillation period and amplitude in a predictable way and with a gene-specific signature on the oscillation amplitude (Fig. 7). Therefore, the global dynamical properties of the repressilator can be tuned simply by changing the parameters relative to a single gene.

\section{Chromosomal position of genes affects the circuit dynamical properties at varying growth rates}

To isolate the effect of genomic position of genes on the dynamics of a chromosomally integrated repressilator, we consider the case of a circuit that is symmetric in terms of intrinsic gene properties, but where a gene is placed at a varying distance from the replication origin. This situation is illustrated in Fig. 8(a), where the genes with protein product concentrations $x_{1}$ and $x_{2}$ are placed in contiguous positions, thus at approximately the same distance $\ell_{12}$ from Ori, while the gene corresponding to $x_{3}$ is in position $\ell_{3}$. In modeling terms, the equivalence of intrinsic gene properties corresponds to the assumption of identical effective basal expression $x_{i} F_{i}(\mu)$ at extremely slow growth, where the growth dependence of gene dosage $g_{i}$ for each gene $i$ is irrelevant since $g_{i} \simeq 1$ for every genomic position. In other words, the following relation is satisfied: $x^{*} F(\mu \simeq 0)=x_{3}^{*} F_{3}(\mu \simeq 0)$, where $x^{*}$ and $F(\mu)$ are the basal expression level and growth-dependence function of genes 1 and 2 , and $F_{3}(\mu)$ differs from $F(\mu)$ only because of the position-dependent scaling of gene dosage with growth rate.

It is straightforward to verify that the dynamics of a repressilator satisfying the above conditions is described by Eq. (8) with $A=2^{\mu C \Delta \ell}$, where $\Delta \ell=\ell_{12}-\ell_{3}$ and $C$ is the time required for DNA replication $(C \simeq 40 \mathrm{~min}$ for fast growth [34]). The factor $A$ is simply the ratio between the gene copy numbers $g_{3} / g_{1(2)}$ as given by the Cooper-Helmstetter model (see the Appendix), and encodes the growth-rate-dependent level of asymmetry induced by gene position in an otherwise symmetrical circuit. Note that the sign of the relative position
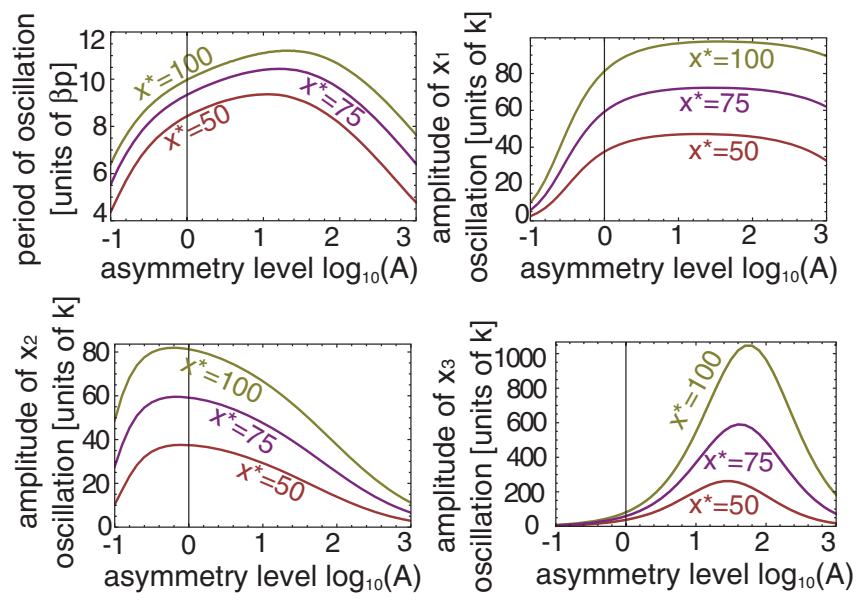

FIG. 7. (Color online) Influence of asymmetry on period and amplitude of oscillation. The figure shows the nonlinear effects of the asymmetry induced by the presence of gene $x_{3}$ with a basal expression level that differs by a factor $A$ from the other genes' basal expression $x^{*}$. The period (upper left plot) and the gene-specific oscillation amplitudes (other plots) are shown as a function of the asymmetry level $\log _{10}(A)$ for three basal expression values that ensure oscillations in the whole range of asymmetry explored. The curves are obtained by measuring period and amplitude of numerical solutions of Eq. (8) for values of $\log _{10}(A)$ spaced by 0.01 in the range $\{-1,3\}$. 
(a)

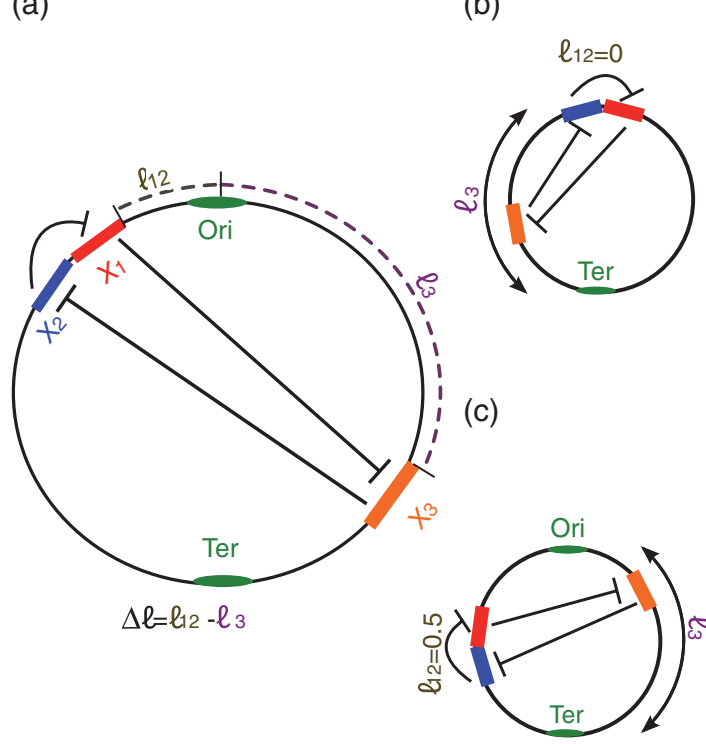

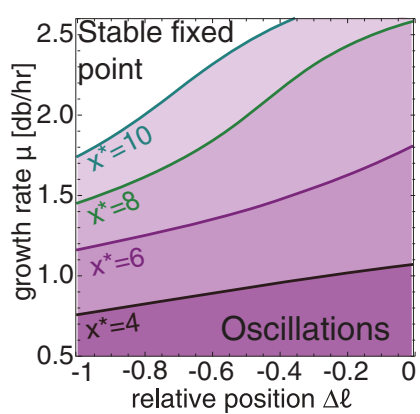

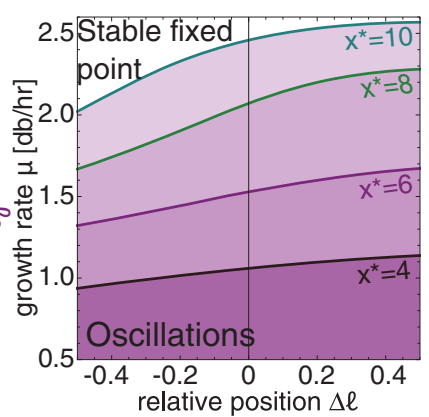

(d)

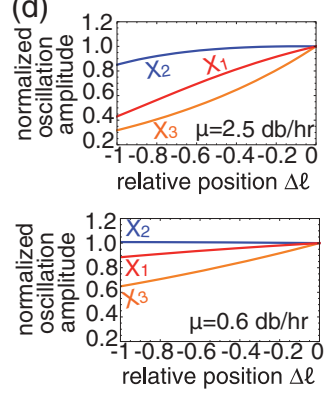

(e)
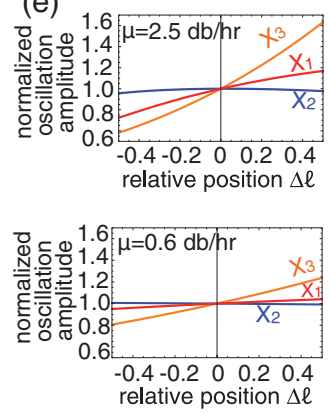

FIG. 8. (Color online) Effect of gene-dosage induced asymmetry on oscillation stability. (a) Scheme of the repressilator chromosomal configuration analyzed here. Two genes are placed at approximately the same distance $\ell_{12}$ from the origin of replication (possibly on opposite arms), while the third is in a different position $\ell_{3}$. This genomic configuration induces a growth-rate-dependent copy-number imbalance between genes, as a consequence of DNA replication. The difference $\Delta \ell=\ell_{12}-\ell_{3}$ measures the effective asymmetry level ( $\Delta \ell=0$ corresponds to the symmetric case) since the ratio between the gene dosages [the factor $A$ in Eq. (8)] is $2^{\mu C \Delta \ell}$, where $C$ is the DNA replication time. (b), (c) The region of oscillation stability is plotted as a function of growth rate $\mu$ and relative position $\Delta \ell$ for different values of the basal expression $x^{*}$. The genomic configurations analyzed, and thus the values of $\Delta \ell$, are obtained moving one gene along the chromosome while keeping the others near the replication origin $\left(\ell_{12}=0\right)$ in plot $(b)$, or in a mid position $\left(\ell_{12}=0.5\right)$ in plot (c) (see the corresponding schemes on the left). The plots show how gene positions influence the range of growth rates in which stable oscillations take place. (d), (e) Effect of the relative position $\Delta \ell$ on the oscillation amplitude, normalized with its value in the symmetric case, of the three proteins is plotted for two different growth rates (the basal expression is $x^{*}=75$ in units of $k$ ). The sets of positions considered in (d) and (e) are those shown in plots (b) and (c), respectively. The change in oscillation amplitude that can be obtained by placing the same genes in different configurations depends on the growth conditions.

$\Delta \ell$ has relevance. For example, the configuration with two genes near the replication origin and the third near the terminus $(\Delta \ell=-1)$ leads to a quantitatively different dynamics with respect to the opposite configuration with $\Delta \ell=1$. The repressilator dynamics can be analyzed for different values of $\Delta \ell$ to explore the effects of gene position.

Figure 8(b) shows a stability diagram obtained moving one gene along the chromosome while the other two genes are near the replication origin $\left(\ell_{12}=0\right)$, for different levels of basal expression $x^{*}$. Analogously, the stability diagram in Fig. $8(\mathrm{c})$ is obtained keeping $\ell_{12}=0.5$ and varying the third gene position $\ell_{3}$. Interestingly, this analysis shows that at fixed growth rate, the repressilator can converge to a limit cycle or to oscillations depending on where genes are integrated on the chromosome, highlighting the importance of including the chromosomal gene coordinates as variables in genetic circuit models. The positional effect is even more evident if two gene positions are varied (data not shown).

As discussed in the previous section, the oscillation stability can be reinforced by increasing the basal expression of one gene. A simple way to increase the basal expression of one gene for a chromosomally integrated circuit is moving its position toward Ori, since this increases its average gene copy number. Indeed, gene position has been used to modulate the oscillation features in an experimental synthetic implementation of another genetic circuit [35]. However, as we show for our case, the effect of the displacement of a gene is growth-rate dependent. In fact, while moving a gene from Ter to Ori allows an increment of its gene dosage of a factor 4 for a $\mu=3 \mathrm{db} / \mathrm{hr}$, this factor decreases with doubling time up to 1 for slow growth. The consequence of this observation on the repressilator dynamics is evident looking at Figs. 8(b) and $8(\mathrm{c})$. The same change in configuration (change in $\Delta \ell$ ) has different effects depending on the growth rate. The modeling framework adopted here gives a quantitative prediction of the gain in oscillation stability that can be achieved by moving the genes along the chromosome depending on the experimental growth conditions, giving an experimental guideline for the best gene insertion sites in order to obtain the desired dynamics. Finally, Figs. 8(d) and 8(e) show the oscillation amplitude dependence on the relative gene positions for the configurations described in Figs. 8(b) and 8(c), respectively. This dependence changes significantly at different growth rates, showing that the effect of gene position is strongly influenced by the cellular environment, and thus by growth rate. This feature is also relevant for synthetic biology since identical experiments carried out with different nutrient levels could in principle lead to different results. 


\section{DISCUSSION}

To sum up, this work addresses the dynamics of a paradigmatic bacterial oscillatory gene circuit, the repressilator, using a modeling framework that accounts for relevant physiological parameters, through their growth-rate dependence [15]. From the modeling viewpoint, this framework entails assuming that the parameters of the dynamical system are in fact dependent on a hidden "superparameter", the growth rate, which encapsulates cell physiology. Additional models or experiments are required in order to obtain the dependence of each relevant parameter from the superparameter. For the case of E. coli, all this information is available. The parameters change their value following the hidden variable, which makes the phenomenology of the dynamical system with respect to the standard parameters less informative. Specifically, ignorance of the role of the superparameter and its behavior prevents from obtaining the physically relevant phase diagram.

A growth-rate induced "dynamical switch". Our results show that the dynamics is dependent on the growth rate in a fashion that is both qualitative and quantitative. Specifically, a symmetric repressilator will lose its oscillatory state with increasing growth rate unless its basal expression is sufficiently high, and this phenomenology is expected to be observable in a wide range of experimentally accessible conditions. Indeed, the growth-mediated switch between different dynamics shown in Fig. 4 should be simple to observe experimentally, given the typical values of the parameters involved. The average protein copy numbers per cell span different orders of magnitude, from $10^{-1}$ to $10^{4}$ [24], while the values for the dissociation constants have been reported to range between a few molecules [36] and a few thousand [37]. With these numbers, the basal expression values (i.e., protein concentration at $\mu=1$ in units of $k$ ) analyzed here, such as $x^{*} \in(0,20)$ in the example in Fig. 4, are well in the physiological range. This suggests that a repressilator dynamics characterized by loss of the oscillatory behavior at a critical growth rate should not be too difficult to observe in the laboratory, and that changes in both oscillation period and amplitude should also be quite likely to be measurable experimentally. ${ }^{3}$

Dependence of the dynamics on the physical support. Furthermore, the growth-rate-dependent behavior of the circuit is affected by the support it is embedded in, a plasmid or a chromosome, and for a chromosome on the detailed coordinates of the three promoters. Figures 4 and 5 suggest that the stability of oscillatory behavior is more sensitive to variations in growth rate for a repressilator integrated on plasmids than on the chromosome, as plasmid copy number can be strongly growth-rate dependent (as well as variable from cell to cell). However, integration on a high-copy-number plasmid can naturally increase the protein concentration (hence, the "promoter strength" parameter defined by Klumpp and coworkers), thus leading to more robust oscillations. Therefore, if the goal is engineering a stable synthetic oscillator, there

\footnotetext{
${ }^{3}$ Note, however, that some of the dissociation constant values reported in the literature were measured in vitro and thus could be underestimated with respect to in vivo values, where nonspecific binding plays an important role.
}

is probably a tradeoff between the advantage of an increased basal expression typical of a high-copy-number plasmid, and the unavoidable plasmid-specific growth-rate dependence (and cell-to-cell variability) of the copy number [38].

On the other hand, for chromosomally integrated repressilators, the dynamics depends nontrivially on gene position. Recently, it has been shown that the spatial ordering of a set of "important" genes along the chromosome is strongly conserved between different bacterial species and largely corresponds to their expression pattern during growth [39], pointing to a functional role for gene chromosomal position. The example of a putative chromosome-integrated repressilator analyzed here suggests that the dynamics of genetic networks, in fast-growing bacteria, should be influenced by the genomic position of its components. For example, for the repressilator case (Fig. 8), a variation in the relative position of genes involved in a regulatory circuit can have different consequences depending on the growth rate. In this perspective, the analysis of the phenotypic consequences of chromosomal rearrangements, such as large inversions [40], should be revisited taking into account the growth conditions. More generally, it is tempting to speculate that the evolutionary pressure for keeping a certain gene order with respect to genome replication may be partially due to natural selection of specific network dynamics defined by the combination of gene positions and cell growth state.

Role of noise. Some relevant considerations can be made about the possible role of noise in the results given here within a purely deterministic framework. In general, noise can strongly affect the dynamics of a repressilator. For example, in the in vivo realization of the repressilator [14], only about $40 \%$ of the cells displayed oscillations, with high cell-to-cell variability in oscillation period and amplitude. Several studies have analyzed the possible impact of noise, focusing on the stochasticity that arises from the discrete nature of the molecular players and from the inherent randomness of their interactions (together referred to as intrinsic noise) [14,29,41]. The main result is that intrinsic noise can both play a constructive role in oscillation robustness and a destructive one. The constructive phenomenon can enlarge the parameter space of oscillations through a resonance effect [41]. The destructive one causes strong cell-to-cell variability in oscillation amplitude and period [14].

While intrinsic noise can be a relevant factor and could partially explain the experimentally observed variability, the dominant source of noise might be due to fluctuations in global cellular parameters (extrinsic noise), such as ribosome or polymerases concentration. This has been shown to be the case in $E$. coli, for relatively high expression (more than approximately 10 proteins per cell) [24]. Since oscillations in the repressilator generally require strong promoters (high basal expressions), the variability in the circuit dynamics is expected to be highly sensitive to the extrinsic noise level, which adds up, for plasmids, to the aforementioned cell-tocell copy-number variation. These considerations point to an interest in considering the stochastic aspects of the circuit. However, in order to extend the mean-field model introduced here to analyze the growth-rate dependence of the cell-to-cell variability of the repressilator dynamics, it would be necessary to know how the extrinsic noise scales with growth rate. 
Unfortunately, there are no experimental data concerning this scaling, making the extension of this work to the stochastic case premature.

Nevertheless, the possibility of a switch between different dynamical regimes in response to the physiological cell state opens interesting considerations about the robustness of this oscillatory genetic circuit. Fluctuations in physiological parameters such as the growth rate fall in the broadly defined category of extrinsic noise. It has been suggested that fluctuations in the growth rate, mainly through its influence on protein dilution, can account for a considerable part of the measured extrinsic noise $[27,42]$. Our analysis suggests that fluctuations in cell parameters linked to growth can introduce cell-to-cell variability in the circuit dynamics (convergence to oscillation or to a stable fixed point) as well as in oscillation period and amplitude. Therefore, the reasons behind the lack of robustness of the repressilator realized in vivo [14] should be also searched in the variability of physiological parameters rather than focusing exclusively on intrinsic noise effects.

Biological outlook. Finally, we believe these findings could be relevant from both a systems biology and a synthetic biology perspective. There is a long list of endogenous oscillators in bacteria [21] that are interesting for the former discipline, and need to be understood within the framework adopted here. The most important examples are circadian clocks and the cell cycle itself. We previously studied the dynamics of the DnaA ${ }^{4}$ oscillatory circuit, which is determinant in this last process [23]. In this case, the time scale of oscillations matches (by definition) the cell-cycle time, thus the approximations defined in Sec. II A are not valid. More complex models are required, and there is no commonly accepted theoretical framework to describe this case. However, it is interesting to note that in the simple modeling framework adopted here, the period of oscillation decreases with growth rate without the need of specific additional regulation. In absence of overlapping replication rounds, this is exactly the kind of behavior desired for an oscillator regulating the triggering of DNA replication, such as the DnaA circuit: a shortening of the initiation time is required when the cell volume grows faster to synchronize DNA replication and volume doubling (the situation becomes more complex at fast growth [23]).

In contrast, circadian clocks need to be resilient to changes in the cell doubling time, and thus in the growth rate, in order to keep a steady $24-\mathrm{h}$ period in variable environmental conditions, and thus can not measure time using the cell cycle. The consequent decoupling between the cell cycle and the circadian rhythm has indeed been verified in cyanobacteria [43-45]. Our results suggest that the dynamics of a genetic oscillator is naturally strongly connected to the cellular growth rate. Therefore, specific regulatory mechanisms are required in order to compensate for these effects and render a circadian oscillator insulated from the growth state. Although circadian clocks appear to be primarily based on post-translational circuits in bacteria [21], the proteins involved are the result

\footnotetext{
${ }^{4} \mathrm{DnaA}$ is a protein responsible for the initiation of DNA replication in several bacteria. Essentially, if present in a sufficiently high concentration, it can promote the melting of DNA strands at the replication origin.
}

of a gene expression process, and thus in principle coupled with growth rate [15]. It would be interesting to evaluate experimentally if the promoters regulating these proteins are more buffered as a function of growth rate compared to others. Quantitative models taking into account the parameter dependence on growth rate, such as the one presented here, may be important to pose the question of the growth-rate robustness of the circadian cycle. For example, the circuit architectures and the type of regulations selected by evolution to compose circadian oscillators might be, at least in part, constrained by the implementation of the observed growth-rate independence.

Finally, from a synthetic biology standpoint, changing the conditions in which the cells are grown alters quantitatively the characteristics of the repressilator dynamics in a predictable manner. This offers the possibility of external control of the circuit behavior by simply operating on macroscopic variable related to physiology such as the type of nutrient supply or the temperature. This way, the engineering and control of the dynamics can be performed by tuning environmental conditions in a model-guided way, rather then by modifying the genetic components, which can be technically complex.

\section{ACKNOWLEDGMENTS}

We acknowledge useful discussions with V. F. Scolari, M. Zarei, and B. Sclavi. We thank M. A. A. Grant and C. Bosia for critical reading of the manuscript. This work was supported by the the International Human Frontier Science Program Organization (Grant No. RGY0069/2009-C).

\section{APPENDIX: POPULATION AVERAGES VERSUS CELL-CYCLE AVERAGES}

A common assumption in genetic circuit modeling is that the contribution of the cell-cycle dynamics can be neglected, at least when one is interested in time scales longer than the doubling time, which is often the case given the typical high protein stability. This approximation allows the use of effective parameters obtained averaging over the cell cycle. The contribution of protein dilution due to growth and division is incorporated as an effective degradation rate [27]. However, as discussed in the text, the growth-rate dependencies of gene expression parameters are derived from experimental observations of average cellular properties in a population [16], which are also affected by the cell age distribution. A quantitative estimate of the age-structure effects on measurements performed on an exponentially growing population is especially important when their growth rate dependence is in analysis. In fact, the fraction of cells found at a certain cell-cycle stage is itself a function of the growth rate.

This appendix discusses the quantitative difference between population and cell-cycle averages for two quantities that are well characterized: the gene dosage due to DNA replication and the cellular volume. We will show that in these two cases, population averages can be used in dynamic models of genetic circuits without introducing significant errors. The assumption that this result can be generalized 
to other quantities justifies, although not rigorously, the use of available experimentally estimated population averages in dynamic models for those quantities whose time dependence (or even cell-cycle averages) are not known [15]. This is the case for ribosome or polymerase concentrations at different growth rates, which are crucial to determine the growthrate dependence of transcription and translation rates in Eq. (3).

Thus, in our case, given the small quantitative difference between the two type of averages, the empirical growth-rate dependencies (based on population averages) have been used in the analysis (and thus for all the parameters quoted in the main text). The alternative use of cell-cycle averages is possible only for the gene dosage and volume, and does not alter significantly the results. Indeed, as reported in [27], for a constitutive gene the average protein concentrations calculated with respect to an age-structured population or with respect to the cell cycle differ by only a few percent.

\section{Cooper-Helmstetter model and gene dosage}

DNA replication in fast-growing bacteria such as E. coli typically starts from a single replication origin (Ori) and proceeds bidirectionally along the circular chromosome until it reaches the replication terminus (Ter). The CooperHelmstetter model [22] establishes the relation between growth rate and replication timing such that DNA copies are produced on time for each newborn cell. The model is based on the empirical observation that the time necessary for chromosome replication (called " $C$ period") and the time period between completion of chromosome replication and the following cell division ( $D$ period) are approximately constant (at least for fast-dividing cells [34], with doubling times less than $1 \mathrm{~h}$ ). Since at time $C+D$ the cell divides, a time lag $X$ before initiation is necessary to make the total replication time $X+C+D$ an integer multiple of the doubling time $\tau$, "synchronizing" DNA replication and cell division. Thus, the following relation has to be satisfied:

$$
X+C+D=(n+1) \tau,
$$

where $n=\operatorname{Int}\left[\frac{C+D}{\tau}\right]$ is the integer number of times that $\tau$ divides $C+D$. Starting from this relation, it can be easily shown that the number of origins present at initiation is exactly $2^{n}$ [16,22]. More generally, we can consider a gene at a chromosomal position defined by its normalized distance $\ell$ from Ori, i.e., $\ell=0$ represents a gene in Ori and $\ell=1$ in Ter. The copy number of this gene $g$ changes during the cell cycle following

$$
g(t)=\left\{\begin{array}{lll}
2^{n^{\prime}} & \text { if } \quad 0<t<\left(n^{\prime}+1\right) \tau-(C(1-\ell)+D), \\
2^{n^{\prime}+1} & \text { if } \quad\left(n^{\prime}+1\right) \tau-(C(1-\ell)+D)<t<\tau,
\end{array}\right.
$$

where $n^{\prime}=\operatorname{Int}\left[\frac{C(1-\ell)+D}{\tau}\right]$. Therefore, the gene dosage averaged over the cell cycle (which could be measured following a single cell lineage and averaging over time) is given by

$$
\begin{aligned}
\langle g(t)\rangle_{\text {cell cycle }} & =\frac{1}{\tau} \int_{0}^{\tau} g(t) d t \\
& =2^{n^{\prime}}\left\{1-n^{\prime}+\mu[C(1-\ell)+D]\right\} .
\end{aligned}
$$

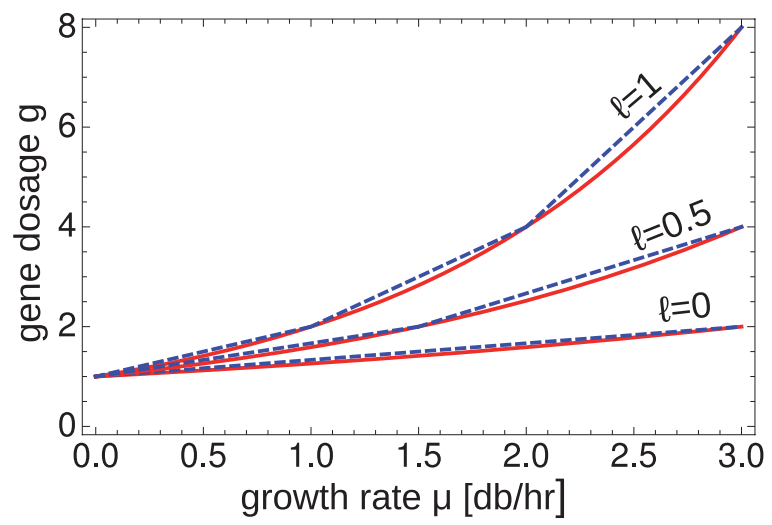

FIG. 9. (Color online) Cell cycle and population average of gene dosage. The gene dosage $g$ averaged over the cell cycle (dashed blue lines) and averaged over a population in balanced exponential growth (continuous red lines) are shown as a function of the growth rate for different chromosomal positions $\ell$ (normalized distance from Ori). In the physiological range of growth rates, the two quantities do not present a substantial quantitative difference.

On the other hand, the population age structure must be taken into account when evaluating the average gene dosage in a cell population. For ideal "balanced exponential" growth with rate $\mu$, this distribution is given by $[27,46]$

$$
a(t, \mu)=2 \ln 2 \mu 2^{-\mu t} .
$$

Thus, the population average is

$$
\langle g(t)\rangle_{\text {population }}=\int_{0}^{\tau} a(t, \mu) g(t) d t=2^{\mu[C(1-\ell)+D]} .
$$

This is the expression typically used to evaluate the gene dosage $[15,16]$. As shown in Fig. 9, the difference between cell-cycle averages (dashed blue lines) and population averages (red continuous lines) in the physiological range of growth rates is negligible for all gene positions.

\section{Cell volume growth}

Similar considerations can be carried out in the case of the average cell volume. The functional form of the volume increase in time during a cell cycle has long been debated [47], with two prevailing hypotheses of linear growth (constant rate) or exponential growth (size-dependent rate), although more complex dependencies have been proposed [48]. Recent experiments strongly suggest an exponential growth [49], and we assumed this functional form (note that the same reasoning could be applied to linear growth straightforwardly, so this choice has no consequences on any of the results). With a volume growth of the form $V(t)=V_{0} 2^{\mu t}$, the mean volume over a cell cycle is $\langle V(t)\rangle_{\text {cell cycle }}=V_{0} / \ln 2$, while the integration over the population leads to $\langle V(t)\rangle_{\text {population }}=$ $2 \ln 2 V_{0}$. All the volume growth-rate dependence is hidden in $V_{0}$, and experimental results indicate that this dependence is approximately exponential [25]. For all the situations considered in our study, we verified that the different numerical factors introduced by averaging over the cell cycle or over the population do not affect significantly the growth-rate dependence of the mean volume. 
[1] A. Goldbeter, Biochemical Oscillations and Cellular Rhythms (Cambridge University Press, Cambridge, UK, 1997).

[2] A. Goldbeter, Nature (London) 420, 238 (2002).

[3] J. J. Tyson, R. Albert, A. Goldbeter, P. Ruoff, and J. Sible, J. R. Soc. Interface 5(Suppl. 1), S1 (2008).

[4] O. Purcell, N. J. Savery, C. S. Grierson, and M. di Bernardo, J. R. Soc. Interface 7, 1503 (2010).

[5] L. Bintu, N. E. Buchler, H. G. Garcia, U. Gerland, T. Hwa, J. Kondev, and R. Phillips, Curr. Opin. Genet. Dev. 15, 116 (2005).

[6] U. Alon, An Introduction to Systems Biology: Design Principles of Biological Circuits (Chapman \& Hall/CRC Press, Boca Raton, FL, 2006).

[7] M. E. Wall, W. S. Hlavacek, and M. A. Savageau, Nat. Rev. Genet. 5, 34 (2004).

[8] G.-W. Li and X. S. Xie, Nature (London) 475, 308 (2011).

[9] E. Andrianantoandro, S. Basu, D. K. Karig, and R. Weiss, Mol. Syst. Biol. 2, 2006.0028 (2006).

[10] N. J. Guido, X. Wang, D. Adalsteinsson, D. McMillen, J. Hasty, C. R. Cantor, T. C. Elston, and J. J. Collins, Nature (London) 439, 856 (2006).

[11] J. Haseloff and J. Ajioka, J. R. Soc. Interface 6(Suppl. 4), S389 (2009).

[12] N. Nandagopal and M. B. Elowitz, Science 333, 1244 (2011).

[13] W. Weber and M. Fussenegger, Nat. Rev. Genet. 13, 21 (2012).

[14] M. B. Elowitz and S. Leibler, Nature (London) 403, 335 (2000).

[15] S. Klumpp, Z. Zhang, and T. Hwa, Cell 139, 1366 (2009).

[16] H. Bremer and P. Dennis, in Escherichia coli and Salmonella, edited by F. C. Neidhardt (ASM Press, Washington, DC, 1996), pp. $1553-1569$.

[17] M. Scott, C. W. Gunderson, E. M. Mateescu, Z. Zhang, and T. Hwa, Science 330, 1099 (2010).

[18] M. Scott and T. Hwa, Curr. Opin. Biotechnol. 22, 559 (2011).

[19] C. Tan, P. Marguet, and L. You, Nat. Chem. Biol. 5, 842 (2009).

[20] R. Kwok, Nature (London) 463, 288 (2010).

[21] P. Lenz and L. Søgaard-Andersen, Nat. Rev. Microbiol. 9, 565 (2011).

[22] S. Cooper and C. Helmstetter, J. Mol. Biol. 31, 519 (1968).

[23] M. A. A. Grant, C. Saggioro, U. Ferrari, B. Bassetti, B. Sclavi, and M. C. Lagomarsino, BMC Syst. Biol. 5, 201 (2011).

[24] Y. Taniguchi, P. J. Choi, G.-W. Li, H. Chen, M. Babu, J. Hearn, A. Emili, and X. S. Xie, Science 329, 533 (2010).

[25] M. Schaechter, O. Maaloe, and N. O. Kjeldgaard, J. Gen. Microbiol. 19, 592 (1958).
[26] S. Klumpp and T. Hwa, Proc. Natl. Acad. Sci. USA 105, 20245 (2008).

[27] R. Marathe, V. Bierbaum, D. Gomez, and S. Klumpp, J. Stat. Phys. 148, 607 (2012).

[28] S. Müller, J. Hofbauer, L. Endler, C. Flamm, S. Widder, and P. Schuster, J. Math. Biol. 53, 905 (2006).

[29] N. Strelkowa and M. Barahona, J. R. Soc. Interface 7, 1071 (2010).

[30] O. Buse, R. Prez, and A. Kuznetsov, Phys. Rev. E 81, 066206 (2010).

[31] R. Blossey and C. V. Giuraniuc, Phys. Rev. E 78, 031909 (2008).

[32] S. Klumpp, PLoS One 6, e20403 (2011).

[33] H. E. Samad, D. D. Vecchio, and M. Khammash, in Proceedings of the 2005 American Control Conference, American Automatic Control Council, 2005, pp. 4405-4410.

[34] O. Michelsen, M. J. T. de Mattos, P. R. Jensen, and F. G. Hansen, Microbiology (Reading, UK) 149, 1001 (2003).

[35] M. R. Atkinson, M. A. Savageau, J. T. Myers, and A. J. Ninfa, Cell 113, 597 (2003).

[36] L. Bintu, N. E. Buchler, H. G. Garcia, U. Gerland, T. Hwa, J. Kondev, T. Kuhlman, and R. Phillips, Curr. Opin. Genet. Dev. 15, 125 (2005).

[37] Y. Setty, A. E. Mayo, M. G. Surette, and U. Alon, Proc. Natl. Acad. Sci. USA 100, 7702 (2003).

[38] S. Tal and J. Paulsson, Plasmid 67, 167 (2012).

[39] P. Sobetzko, A. Travers, and G. Muskhelishvili, Proc. Natl. Acad. Sci. USA 109, E42 (2012).

[40] E. Esnault, M. Valens, O. Espéli, and F. Boccard, PLoS Genet. 3, e226 (2007).

[41] M. Yoda, T. Ushikubo, W. Inoue, and M. Sasai, J. Chem. Phys. 126, 115101 (2007).

[42] S. Tsuru, J. Ichinose, A. Kashiwagi, B.-W. Ying, K. Kaneko, and T. Yomo, Phys. Biol. 6, 036015 (2009).

[43] T. Kondo, T. Mori, N. V. Lebedeva, S. Aoki, M. Ishiura, and S. S. Golden, Science 275, 224 (1997).

[44] T. Mori and C. H. Johnson, J. Bacteriol. 183, 2439 (2001).

[45] I. Mihalcescu, W. Hsing, and S. Leibler, Nature (London) 430, 81 (2004).

[46] E. O. Powell, J. Gen. Microbiol. 15, 492 (1956).

[47] A. L. Koch, Crit. Rev. Microbiol. 19, 17 (1993).

[48] G. Reshes, S. Vanounou, I. Fishov, and M. Feingold, Biophys. J. 94, 251 (2008).

[49] M. Godin, F. F. Delgado, S. Son, W. H. Grover, A. K. Bryan, A. Tzur, P. Jorgensen, K. Payer, A. D. Grossman, M. W. Kirschner et al., Nat. Methods 7, 387 (2010). 